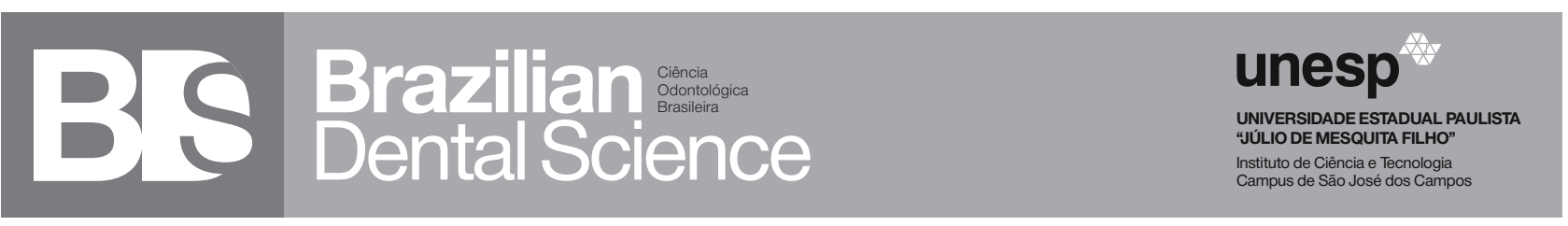

\title{
EDITORIAL
}

\section{LOW TEMPERATURE ATIMOSPHERIC PRESSURE PLASIMA: A PROMISING THERAPY FOR THE FUTURE DENTISTRY}

Dentistry evolved rapidly in the last decades, generating more technological and aesthetic solutions for patients, increasing the efficacy in the promotion of health and life quality. However, big challenges still persist and move the scientific community towards new solutions. Clinical conditions that lead to therapeutic failures, such as periimplantitis, refractory endodontic and periodontal infections, persistent oral candidosis, are part of clinical routine.

In particular, in the context of infectious diseases, a dark scenario comes out - a significative increase in the occurrence of antimicrobial resistance and few new molecules being discovered and effectively marketed. Besides, treating biofilm-associated diseases, such as most of the oral diseases, is challenging. Thus, in order to face these challenges, new therapeutic alternatives are needed.

The search for new alternatives points out the physical plasma. Plasma is called the "fourth state of matter" and is generated by the application of energy, such as electrical field, to a neutral gas. Cold plasma has interesting biological effects that are useful in medical area, such as antimicrobial, anti-inflammatory, anti-neoplasic effects and the ability to induce tissue repair. These biological effects are associated to plasma reactive species, such as neutral oxygen and nitrogen species (ROS and RON).

The inhibitory effects on Gram-positive and Gram-negative bacteria were reported in the literature, showing that plasma has effect on cell wall, cell membrane, and biofilm polymeric matrix. Interestingly, cold plasma showed also inhibitory effect on multi-resistant bacterial strains, increasing the interest on this technology. To the best of our knowledge, there are no reports on the development of resistance to cold plasma so far.
Studies point out the use of cold plasma as adjuvant in the treatment of oral candidosis. The inhibitory effect and action on virulence factors of Candida albicans were reported. The reduction of fungal hyphae epithelial invasion and antiinflammatory effect showed positive effect in the treatment of oral candidosis induced in murine model.

Biofilms associated to endodontic diseases also had significant reduction in viability after treatment with cold plasma, showing higher effectiveness when compared to antimicrobial photodynamic therapy.

Interestingly, cold plasma did not show any cyto or genotoxicity to several cell lines.

Other applications in Dentistry have been suggested and should be highlighted.

The use of cold plasma in combination to low concentration of carbamide peroxide for dental bleaching showed promising results and higher preservation of dental structure when compared to standard treatment.

Lastly, the application of cold plasma to the control of malignant neoplasias has been focused by many studies in medical area and the results are exciting. An interesting study reported that cold plasma was able to inhibit the invasion of oral squamous cells carcinoma due to the reduction in the adhesion and matrix metalloproteinases activity.

There are some cold plasma devices developed and purchased in Europe, mainly focused to the use in Dermatology, and the applications in the medical area are expanding rapidly. The recent findings on cold plasma are promising and exciting and pointing out to a wide application of this technology in Future Dentistry. 


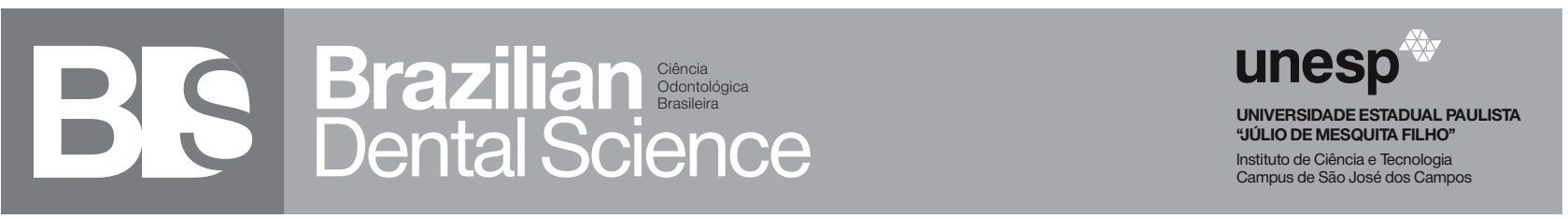

\section{REFERENCES}

Weltman KD et al. The future for plasma science and technology. Plasma Process Polym 2019;16:e1800118.

Laroussi M, Leipold F. Evaluation of the roles of reactive species, heat, and UV radiation in the inactivation of bacterial cells by air plasmas at atmospheric pressure. International Journal of Mass Spectrometry 2004;233:81-6.

Nishime TMC, Borges AC, Koga-Ito CY, Munemasa M, Hein LRO, Kostov KG. Non-thermal atmospheric pressure plasma jet applied to inactivation of different microorganisms. Surf Coatings Technol, v.312, p.19-24,2017.

Borges AC, Nishime TMC, Kostov KG, Lima GMG, Gontijo AVL, Carvalho JNMM, Honda RY, Koga-Ito CY.Cold atmospheric pressure plasma jet modulates Candida albicansvirulence traits. Clinical Plasma Medicine., v.7-8, p.9 - 15, 2017.

$\mathrm{Nam} \mathrm{SH,} \mathrm{Ok} \mathrm{SM,} \mathrm{Kim} \mathrm{GC.} \mathrm{Tooth} \mathrm{bleaching} \mathrm{with} \mathrm{low-temperature} \mathrm{plasma}$ lowers surface roughness and Streptococcus mutans adhesion. Int Endod J. 2018;51(4):479-488.

Lis KA, Kehrenberg C, Boulaaba A, von Köckritz-Blickwede M, Binder S, Li Y, Zimmermann JL, Pfeifer Y, Ahlfeld B. Inactivation of multidrug-resistant pathogens and Yersinia enterocolitica with cold atmospheric-pressure plasma on stainlesssteel surfaces. Int J Antimicrob Agents. 2018;52(6):811-818.

Armand A, Khani M, Asnaashari M, AliAhmadi A, Shokri B. Comparison study of root canal disinfection by cold plasma jet and photodynamic therapy. Photodiagnosis Photodyn Ther. 2019 Jun;26:327-333.

Kang SU, Seo SJ, Kim YS, Shin YS, Koh YW, Lee CM, Yang SS, Lee JS, Moon E, Kang H, Ryeo JB, Lee Y, Kim CH. Comparative Effects of Non-Thermal Atmospheric Pressure Plasma on Migration and Invasion in Oral Squamous Cell Cancer, by Gas Type. Yonsei Med J. 2017 Mar;58(2):272-281.

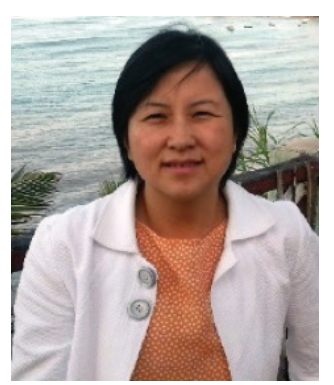

Prof. Dr. Cristiane Yumi Koga Ito

São Paulo State University (Unesp) - Institute of Science and Technology, São José dos campos environmental engineering department - São José dos Campos - SP - Brazil. 


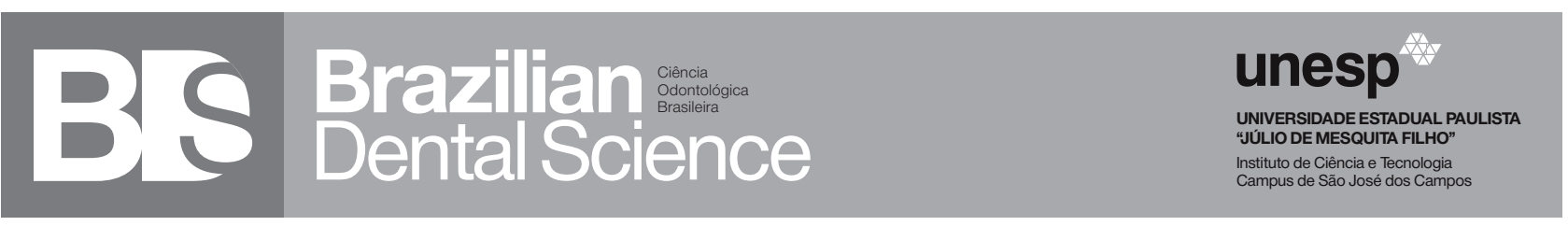

\section{EDITORIAL}

\section{PLASMA FRIO: UIMA TERAPIA PROMISSORA PARA A ODONTOLOGIA DO FUTURO}

A Odontologia evoluiu a passos largos nas últimas décadas, gerando soluções mais tecnológicas e estéticas para os pacientes, aumentando sua eficácia na promoção de saúde e qualidade de vida. No entanto, grandes desafios ainda persistem e fazem a comunidade científica se mover na busca por novas soluções. Condições clínicas que levam ao insucesso terapêutico, tais como periimplantites, infecções endodônticas e periodontais refratárias, candidose oral persistente, são parte da rotina clínica.

Particularmente no contexto das doenças infecciosas, temos um cenário sombrio, de aumento significativo da ocorrência de resistência aos antimicrobianos e poucas novas moléculas sendo descobertas e efetivamente chegando ao mercado. Soma-se a isso, o desafio inerente ao tratamento de doenças biofilme-associadas, como é o caso da maior parte das doenças bucais. Dessa forma, para fazer frente a esses desafios, novas alternativas terapêuticas são necessárias.

Na busca por novas alternativas, surge o plasma físico. O plasma é chamado de "quarto estado da matéria" e é gerado pela aplicação de energia, como uma corrente elétrica, a um gás neutro. O plasma frio apresenta efeitos biológicos de interesse para a área médica, tais como efeito antimicrobiano, antiinflamatório, anti-neoplásico e indutor de reparação tecidual. Esses efeitos biológicos estão relacionados às espécies reativas do plasma, tais como espécies reativas neutras de oxigênio (ROS) e de nitrogênio (RON).

O efeito inibitório do plasma frio sobre bactérias Gram-positivas e Gram-negativas foi reportado na literatura, mostrando que o plasma tem efeito sobre a parede celular e membrana plasmática bacteriana, além da matriz polimérica de biofilmes microbianos. De forma interessante, o plasma frio tem mostrado efeito inibitório sobre cepas bacterianas multirresistentes, o que aumenta ainda mais o interesse por essa tecnologia. Não foram detectados relatos de desenvolvimento de resistência ao plasma frio até o momento.

Estudos tem ainda apontado que o plasma frio pode ser útil como adjuvante no tratamento da candidose bucal. O efeito inibitório e ação sobre fatores de virulência de Candida albicans foram reportados. A redução da invasão epitelial de hifas fúngicas e efeito anti-inflamatório tiveram efeitos positivos no tratamento da candidose bucal induzida em modelo murino.

Biofilmes de interesse endodôntico também tiveram redução significativa na sua viabilidade após tratamento com plasma frio, mostrando efetividade superior ao da terapia fotodinâmica antimicrobiana.

De forma interessante, o plasma frio não tem mostrado cito ou genotoxicidade frente a diversas linhagens celulares.

Outras aplicações na Odontologia também tem sido sugeridas e merecem destaque.

O plasma frio em combinação com concentrações menores de peróxido de carbamida para clareamento dental mostrou resultados promissores e com maior preservação da estrutura dentária em relação ao tratamento padrão.

Por fim, a aplicação do plasma frio para o controle de neoplasias malignas tem sido foco de muitos estudos na área médica e os resultados são empolgantes. Um estudo interessante relatou que o plasma frio inibiu a invasão de células de linhagens de carcinoma espinocelular bucal, graças à redução da adesão e atividade de mataloproteinases da matriz.

Existem alguns equipamentos geradores de plasma frio desenvolvidos e comercializados na Europa, com foco principal na área de Dermatologia, sendo que as aplicações na área médica se expandem rapidamente. Os recentes achados sobre as aplicações do plasma frio são promissores e empolgantes e apontam para uma ampla aplicação dessa tecnologia na Odontologia do Futuro. 


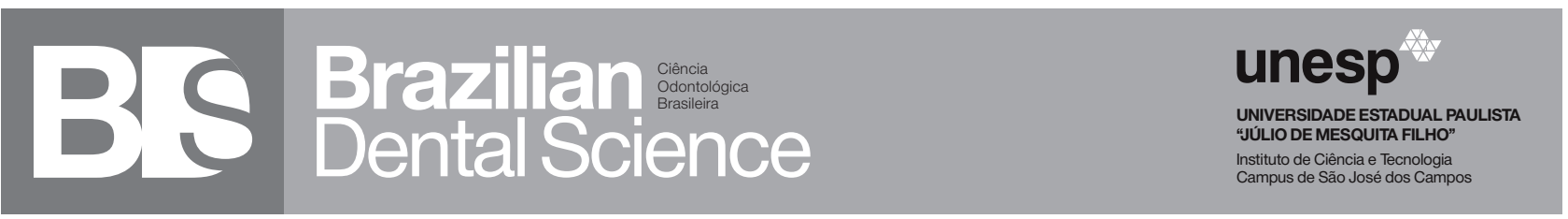

\section{REFERENCES}

Weltman KD et al. The future for plasma science and technology. Plasma Process Polym 2019;16:e1800118.

Laroussi M, Leipold F. Evaluation of the roles of reactive species, heat, and UV radiation in the inactivation of bacterial cells by air plasmas at atmospheric pressure. International Journal of Mass Spectrometry 2004;233:81-6.

Nishime TMC, Borges AC, Koga-Ito CY, Munemasa M, Hein LRO, Kostov KG. Non-thermal atmospheric pressure plasma jet applied to inactivation of different microorganisms. Surf Coatings Technol, v.312, p.19-24,2017.

Borges AC, Nishime TMC, Kostov KG, Lima GMG, Gontijo AVL, Carvalho JNMM, Honda RY, Koga-Ito CY.Cold atmospheric pressure plasma jet modulates Candida albicansvirulence traits. Clinical Plasma Medicine., v.7-8, p.9 - 15, 2017.

$\mathrm{Nam} \mathrm{SH,} \mathrm{Ok} \mathrm{SM,} \mathrm{Kim} \mathrm{GC.} \mathrm{Tooth} \mathrm{bleaching} \mathrm{with} \mathrm{low-temperature} \mathrm{plasma}$ lowers surface roughness and Streptococcus mutans adhesion. Int Endod J. 2018;51(4):479-488.

Lis KA, Kehrenberg C, Boulaaba A, von Köckritz-Blickwede M, Binder S, Li Y, Zimmermann JL, Pfeifer Y, Ahlfeld B. Inactivation of multidrug-resistant pathogens and Yersinia enterocolitica with cold atmospheric-pressure plasma on stainlesssteel surfaces. Int J Antimicrob Agents. 2018;52(6):811-818.

Armand A, Khani M, Asnaashari M, AliAhmadi A, Shokri B. Comparison study of root canal disinfection by cold plasma jet and photodynamic therapy. Photodiagnosis Photodyn Ther. 2019 Jun;26:327-333.

Kang SU, Seo SJ, Kim YS, Shin YS, Koh YW, Lee CM, Yang SS, Lee JS, Moon E, Kang H, Ryeo JB, Lee Y, Kim CH. Comparative Effects of Non-Thermal Atmospheric Pressure Plasma on Migration and Invasion in Oral Squamous Cell Cancer, by Gas Type. Yonsei Med J. 2017 Mar;58(2):272-281.

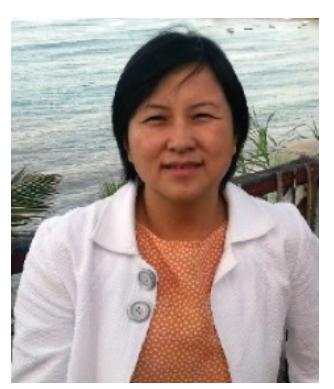

Prof ${ }^{\mathrm{a}}$. Dra. Cristiane Yumi Koga Ito

São Paulo State University (Unesp) - Instituto de Ciencia e Tecnologia, São José dos campos - Departamento de Engenharia Ambiental - São José dos Campos - SP Brazil. 